\title{
On vector-valued modular forms and their Fourier coefficients
}

\author{
by \\ MARvin Knopp (Philadelphia, PA) and \\ Geoffrey Mason (Santa Cruz, CA)
}

1. Introduction. A vector-valued modular form of weight $k$ ( $k$ any real number) on the modular group $\Gamma=\mathrm{SL}(2, \mathbb{Z})$ may be described as follows (see Section 2 for more details): for any integer $p \geq 1$ it is a tuple $\left(F_{1}(\tau), \ldots, F_{p}(\tau)\right)$ of functions holomorphic in the complex upper half-plane $\mathcal{H}$ together with a $p$-dimensional complex representation $\varrho: \Gamma \rightarrow \mathrm{GL}(p, \mathbb{C})$ satisfying the following conditions:

(a) For all $V=\left(\begin{array}{ll}a & b \\ c & d\end{array}\right) \in \Gamma$ we have

$$
\left.\left(F_{1}, \ldots, F_{p}\right)^{\mathrm{t}}\right|_{k} V(\tau)=\varrho(V)\left(F_{1}(\tau), \ldots, F_{p}(\tau)\right)^{\mathrm{t}}
$$

(t refers to transpose of vectors and matrices).

(b) Each function $F_{j}(\tau)$ has a convergent $q$-expansion holomorphic at infinity:

$$
F_{j}(\tau)=\sum_{n \geq 0} a_{n}(j) q^{n / N_{j}}
$$

for positive integers $N_{j}$. (Here and below, $q=\exp (2 \pi i \tau)$.)

The precise meaning of the right slash operator $\left.\right|_{k} V$ in (1) is as follows. Fix a multiplier system $v$ in weight $k$ with respect to $\Gamma$ (there are six choices for each $k$ ) and define $\left.F\right|_{k} V(\tau)=\left.F\right|_{k} ^{v} V(\tau)=v(V)^{-1}(c \tau+d)^{-k} F(V \tau)$. This defines a right action of the group $\Gamma$ on holomorphic functions $F(\tau)$ which extends to a componentwise action on tuples of such functions. See [K1] for more details. In what follows the multiplier $v$ will play no role and we omit it from the notation. Note that as a consequence of (1) and (2), we necessarily have $k \in \mathbb{Q}$.

We might well have allowed our vector-valued modular forms to have poles, in which case one would want to distinguish between meromorphic and entire vector-valued modular forms. As it is, we will consider only vector-

2000 Mathematics Subject Classification: Primary 11F11. 
valued modular forms in the sense of (1) and (2) in the present paper, and we will usually omit the adjective "entire". If each function $F_{j}(\tau)$ satisfies $a_{0}(j)=0$ we say that the vector-valued modular form is cuspidal.

It has been understood for some time that classical holomorphic modular forms of weight $k$ (on a subgroup $\Delta$ of $\Gamma$ ) may be construed as vector-valued modular forms in the above sense, with $p$ equal to the index $|\Gamma: \Delta|$ and $\varrho$ the permutation representation of $\Gamma$ on the cosets of $\Delta$ (cf. [S]). However, there are examples of vector-valued modular forms which do not arise in this manner. The special case in which $\varrho$ is a monomial representation was investigated by the authors in $[\mathrm{KM}]$. The question then arises: to what extent are properties of classical modular forms shared by the more general class of vector-valued modular forms?

The main result of the present paper, treating a question of this nature, is concerned with obtaining growth conditions on the Fourier coefficients $a_{n}(j)$ which are analogous to well known results in the classical case (cf. $[\mathrm{L}],[\mathrm{R}])$. Aside from any intrinsic interest that the main result of the present paper may have, it plays an important role in the proof of some recent theorems in rational conformal field theory (RCFT) established by the second author and Chongying Dong $([\mathrm{DM}])$. The principle of "modular invariance" is well known in RCFT (cf. [Z], [DLM] for example), and while it remains conjecture that the partition functions of RCFT are classical modular forms, it is presently known only that such partition functions afford a vector-valued modular form. (This is discussed in detail in $[\mathrm{KM}]$.) It is this circumstance which animates the present paper. The main result of the paper is as follows:

THEOREM. Let $\left(F_{1}(\tau), \ldots, F_{p}(\tau)\right)$ be a vector-valued modular form of weight $k$ associated to a representation $\varrho$ of $\Gamma$. There is a nonnegative constant $\alpha$ depending only on $\varrho$ such that the Fourier coefficients $a_{n}(j)$ satisfy the growth condition $a_{n}(j)=O\left(n^{k+2 \alpha}\right)$ for every $1 \leq j \leq p$, as $n \rightarrow \infty$. If the vector-valued modular form is cuspidal then $a_{n}(j)=O\left(n^{k / 2+\alpha}\right)$ for every $1 \leq j \leq p$, as $n \rightarrow \infty$.

Our Theorem is thus an analog of the "elementary" estimates for classical modular forms of weight $k$, namely $a_{n}=O\left(n^{k}\right)$ for holomorphic forms and $a_{n}=O\left(n^{k / 2}\right)$ for cusp-forms. The complications which arise from the more general situation at hand are handled by the use of Eichler's important estimates $([\mathrm{E}])$, which the first author has used elsewhere ([K2]). The proof of the Theorem shows that we may take $\alpha$ equal to $\log p\|\varrho(S)\|$ times a constant independent of $\varrho$.

We use the following notation throughout the paper: $S=\left(\begin{array}{cc}0 & -1 \\ 1 & 0\end{array}\right)$ and $T=\left(\begin{array}{ll}1 & 1 \\ 0 & 1\end{array}\right)$ are the usual generators of $\Gamma ; \tau$ denotes an element in $\mathcal{H}, z$ an element in the closure $\mathcal{R}$ of the usual fundamental region for $\Gamma$ in $\mathcal{H}$. Thus $\mathcal{R}$ is bounded by the two vertical lines consisting of points with real parts 
equal to $1 / 2$ and $-1 / 2$ respectively, and by the arc of the unit circle lying between these lines and in $\mathcal{H}$. We also set $y=\operatorname{Im} \tau$ and $v=\operatorname{Im} z$.

2. Vector-valued modular forms. Let Hol denote the space of holomorphic functions on $\mathcal{H}$ and let $\mathrm{Hol}_{q}$ denote the subspace of those functions which have a $q$-expansion convergent at infinity. Note that Hol is a right $\Gamma$-module with respect to the action $\left.\right|_{k}$, whereas $\mathrm{Hol}_{q}$ is not preserved by the action of $\Gamma$. We write $\mathrm{Hol}_{k}$ when we want to think of $\mathrm{Hol}$ as a right $\Gamma$-module in this way. It is convenient to refer to the pair $(F, \varrho)$ as a vectorvalued modular form if $F=\left(F_{1}(\tau), \ldots, F_{p}(\tau)\right)$ and $\varrho$ is a representation of $\Gamma$ in $\operatorname{GL}(p, \mathbb{C})$ such that $(1)$ and $(2)$ are satisfied. In this case it is evident from (1) that the component functions $F_{j}(\tau)$ span a finite-dimensional $\Gamma$-submodule $M \subseteq \mathrm{Hol}_{k}$; (2) tells us that $M$ is actually contained in $\mathrm{Hol}_{q}$. We are going to show that the converse is also true. Precisely, we have the

Proposition. Let $M \subseteq \mathrm{Hol}_{k}$ be a finite-dimensional $\Gamma$-module which is contained in $\mathrm{Hol}_{q}$, and let $\left(F_{1}(\tau), \ldots, F_{p}(\tau)\right)$ be a tuple of elements in $M$ which generate $M$ as a linear space (the $F_{j}(\tau)$ are not required to be linearly independent). Then there is a representation $\varrho: \Gamma \rightarrow \mathrm{GL}(p, \mathbb{C})$ such that $(F, \varrho)$ is a vector-valued modular form of weight $k$.

The Proposition tells us that the data supplied by a vector-valued modular form is essentially equivalent to that of a finite-dimensional $\Gamma$-submodule $M$ of $\mathrm{Hol}_{k}$ contained in $\mathrm{Hol}_{q}$ together with a tuple of generators of $M$. Given two vector-valued modular forms $(F, \varrho)$ and $\left(F^{\prime}, \varrho^{\prime}\right)$, we say that they are equivalent if they correspond to the same $\Gamma$-module, say $M$. In other words, the component functions of both $F$ and $F^{\prime}$ generate $M$ as a linear space. Note that the representations $\varrho, \varrho^{\prime}$ play a passive role in this. For instance, it is easy to find examples of vector-valued modular forms $(F, \varrho),\left(F^{\prime}, \varrho^{\prime}\right)$ with the same tuple of holomorphic functions and inequivalent representations $\varrho, \varrho^{\prime}$ of $\Gamma$. By way of example, let $F$ and $F^{\prime}$ each consist of the 2 -component vector $(\Delta(\tau), \Delta(\tau))$ where $\Delta(\tau)$ is the usual discriminant function, a cusp-form of weight 12 on $\Gamma$. Take $\varrho$ to be the 2 -dimensional representation in which each element of $\Gamma$ is represented by the identity, and $\varrho^{\prime}$ the 2-dimensional representation whose kernel is the (unique) subgroup of $\Gamma$ of index 2 and such that $S$ interchanges components of $F$.

Proof of the Proposition. For each index $j, 1 \leq j \leq p$, and each $V \in \Gamma$ we have

$$
\left.F_{j}\right|_{k} V(\tau)=\sum_{m=1}^{p} \varrho_{j m}(V) F_{m}(\tau)
$$

for scalars $\varrho_{j m}(V)$. Define a matrix $\left(\varrho_{j m}(V)\right)$. If $\varrho$ defines a representation of $\Gamma$ in $\operatorname{GL}(p, \mathbb{C})$ then $(F, \varrho)$ will be a vector-valued modular form as de- 
sired. It is easy to see that if the component functions $F_{j}(\tau)$ are linearly independent then this is indeed the case. It will not be so in general, however: if the $F_{j}(\tau)$ are linearly dependent then the choice of scalars in (3) is not unique and a "bad" choice will lead to the failure of $\varrho$ to be a representation. We must explain how one may always choose the scalars $\varrho_{j m}(V)$ in (3) so that, regardless whether the $F_{j}(\tau)$ are linearly independent, $\varrho$ will be a representation of $\Gamma$.

In order to simplify notation in the argument to follow we assume (as we may without loss of generality) that the first $d$ component functions $F_{1}(\tau), \ldots, F_{d}(\tau)$ constitute a basis of $M$, and set $G=\left(F_{1}(\tau), \ldots, F_{d}(\tau)\right)$. Thus if $\nu$ is the matrix representation of $\Gamma$ on $M$ then we know from remarks in the preceding paragraph that $(G, \nu)$ is a vector-valued modular form of weight $k$ :

$$
\left.G^{\mathrm{t}}\right|_{k} V(\tau)=\nu(V) G^{\mathrm{t}}
$$

Set $f=p-d$, so that for $f+1 \leq j \leq p$ the functions $F_{j}(\tau)$ are linear combinations of $F_{1}(\tau), \ldots, F_{d}(\tau)$. So there is an $f \times d$ matrix $Q$ such that

$$
\left(F_{d+1}(\tau), \ldots, F_{p}(\tau)\right)^{\mathrm{t}}=Q G^{\mathrm{t}} .
$$

Introduce the $p \times d$ matrix $P=\left(\begin{array}{c}I_{d} \\ Q\end{array}\right)$, the $p \times p$ matrix $R$ (row operations) which satisfies $R P=\left(\begin{array}{c}I_{d} \\ O\end{array}\right)$, and the $p$-dimensional representation of $\Gamma$ given by

$$
\varrho(V)=R^{-1}\left(\begin{array}{cc}
\nu(V) & O \\
O & I_{f}
\end{array}\right) R,
$$

where $I_{n}$ denotes the $n \times n$ identity matrix. Now calculate

$$
\left.F^{\mathrm{t}}\right|_{k} V(\tau)=\left.\left(P G^{\mathrm{t}}\right)\right|_{k} V(\tau)=P \nu(V) G^{\mathrm{t}}=\varrho(V) P G^{\mathrm{t}}=\varrho(V) F^{\mathrm{t}} .
$$

This shows that $(F, \varrho)$ is indeed a vector-valued modular form of weight $k$, and completes the proof of the Proposition.

We define the level of the vector-valued modular form $(F, \varrho)$ to be the least common multiple of the integers $N_{j}$ which occur in (2). As long as $N_{j}$ is always taken to be as small as possible in (2), it is easily seen that equivalent vector-valued modular forms have the same level, so that the level depends only on the equivalence class of vector-valued modular forms to which $(F, \varrho)$ belongs. If $(F, \varrho)$ has level $N$ then $T^{N}$ acts as the identity on the subspace of Hol spanned by the components of $F$, i.e., with previous notation we have $\varrho\left(T^{N}\right)=I$. As a result, the matrix $\varrho(T)$ has finite order and consequently may be diagonalized. This means that we can choose a basis $B_{1}(\tau), \ldots, B_{d}(\tau)$ in such a way that

$$
B_{j}(\tau)=q^{n_{j} / N_{j}} \sum_{n \geq 0} a_{n}(j) q^{n}
$$


for integers $n_{j} \geq 0$. We may rephrase this in the following manner: a vectorvalued modular form is equivalent to a vector-valued modular form $(B, \mu)$ in which the component functions $B_{j}(\tau)$ of $B$ are linearly independent and have $q$-expansions (4). We say that a vector-valued modular form is reduced if it satisfies these conditions. So every vector-valued modular form is equivalent to one which is reduced, and if $(B, \mu)$ is a reduced vector-valued modular form of level $N$ then $\mu(T)$ is a diagonal matrix with diagonal entries which are $N$ th roots of unity.

3. Proof of the Theorem. In proving the Theorem we may replace the given vector-valued modular form $(F, \varrho)$ by any other vector-valued modular form to which it is equivalent. After remarks in the last section we may therefore assume that $(F, \varrho)$ is reduced. Bearing in mind our notational conventions we introduce, following Hecke, the function

$$
g_{j}(\tau)=y^{\sigma}\left|F_{j}(\tau)\right|,
$$

where $\sigma$ is a real constant to be chosen later. Note that $g_{j}(\tau)$ is invariant under the action of $T$. Thanks to $(2)$ there is a constant $K$ such that

$$
g_{j}(\tau)<K v^{\delta \sigma}
$$

for each $j$ and all $z \in \mathcal{R}$, where the constant $\delta$ is equal to 1 or 0 according as $F$ is holomorphic or cuspidal. For $V=\left(\begin{array}{ll}a & b \\ c & d\end{array}\right) \in \Gamma$ we have

$$
g_{j}(V z)=\left(v|c z+d|^{-2}\right)^{\sigma}\left|F_{j}(V z)\right|=v^{\sigma}|c z+d|^{k-2 \sigma}\left|F_{j}\right|_{k} V(z) \mid .
$$

Hence

$$
g_{j}(V z) \leq|c z+d|^{k-2 \sigma} \sum_{m=1}^{p}\left|\varrho_{j m}(V)\right| g_{m}(z),
$$

where we have used the triangle inequality in the last step. Note that if we set $G=\left(g_{1}(z), \ldots, g_{p}(z)\right)$ then the sum in (6) is simply the $j$ th entry of the vector $\left(\left|\varrho_{j m}(V)\right|\right) G^{\mathrm{t}}$.

Next we introduce the Eichler length of $V$ (cf. [E], [K2]) with respect to the generators $S, T$ of $\Gamma$. Namely, we write $V$ as a product

$$
V= \pm V_{1} \ldots V_{L}
$$

where each $V_{j}$ is equal to either $S$ or $T^{n_{j}}$ for some integer $n_{j}$, no two consecutive $V_{j}$ are both equal to $S$ or a power of $T$, and where $L$ is minimal. Then $L=L(V)$ is called the Eichler length of $V$. We have the basic estimate ([E, Theorem 1])

$$
L(V) \leq n_{1} \log \mu(V)+n_{2}
$$

where $\mu(V)=a^{2}+b^{2}+c^{2}+d^{2}$ for $V=\left(\begin{array}{ll}a & b \\ c & d\end{array}\right)$ and $n_{1}, n_{2}$ are constants independent of $V$. Now we have $\varrho(V)=\varrho\left(V_{1}\right) \ldots \varrho\left(V_{L}\right)$, so that

$$
\left|\varrho_{j m}(V)\right| \leq \sum\left|\varrho_{j m_{1}}\left(V_{1}\right)\right|\left|\varrho_{m_{1} m_{2}}\left(V_{2}\right)\right| \ldots\left|\varrho_{m_{L-1} m}\left(V_{L}\right)\right|
$$


where the sum ranges over all indices $1 \leq m_{1}, \ldots, m_{L-1} \leq p$. Introduce a constant $K_{1}$ which satisfies

$$
\left|\varrho_{l m}(S)\right| \leq K_{1}
$$

for all $l, m$. Now if $V_{j}=T^{n_{j}}$ then $\left|\varrho_{l m}\left(V_{j}\right)\right|=\delta_{l, m}$ (Kronecker delta) because our vector-valued modular form is reduced. Since there are just $p^{L-1}$ summands in (8), it follows that

$$
\left|\varrho_{j m}(V)\right| \leq p^{L(V)-1} K_{1}^{L(V)}
$$

for all $1 \leq j, m \leq p$ and all $V \in \Gamma$. Using (5) and (6) we conclude from (9) that

$$
g_{j}(V z)<|c z+d|^{k-2 \sigma}\left(p K_{1}\right)^{L(V)} K v^{\delta \sigma} .
$$

Making use of (7) we obtain

$$
g_{j}(V z)<K_{2}|c z+d|^{k-2 \sigma} \mu(V)^{\alpha} v^{\delta \sigma}
$$

for all indices $j$, all $z \in \mathcal{R}$ and $V \in \Gamma$. Here $K_{2}$ is a constant depending on $\sigma$ but independent of $j, z$ and $V$, while $\alpha=n_{1} \log p K_{1}$ is a constant independent of $\sigma, j, z$ and $V$.

We interpolate a result from [K2, Lemma 6]. There is a choice for the set $\mathcal{M}$ of left coset representatives of $\langle T\rangle$ in $\Gamma$ such that the following holds: there is a constant $K_{3}$ satisfying $\mu(V) \leq K_{3}\left(c^{2}+d^{2}\right)$ for all $M \in \mathcal{M}$. Here, $(c, d)$ is the bottom row of $M$. As a result, if we take $V=T^{n} M$ with $M \in \mathcal{M}$ in (11) then

$$
g_{j}(V z)=g_{j}(M z) \leq K_{4}|c z+d|^{k-2 \sigma}\left(c^{2}+d^{2}\right)^{\alpha} v^{\delta \sigma}
$$

for a constant $K_{4}$ depending only on $\sigma$. We also have

$$
c^{2}+d^{2} \leq|c z+d|^{2} \frac{\left(1+4|z|^{2}\right)}{v^{2}} \leq \frac{20}{3}|c z+d|^{2}
$$

where we used Lemma 4 of [K2] for the first inequality and $|z|^{2} \leq 1 / 4+v^{2}$, $v^{2} \geq 3 / 4$ for the second. Combine (12) and (13) to obtain

$$
g_{j}(V z) \leq K_{5}|c z+d|^{k+2(\alpha-\sigma)} v^{\delta \sigma}
$$

with a constant $K_{5}$ depending only on $\sigma$. We now pick $\sigma=\alpha+k / 2$, as we may because $\alpha$ is independent of $\sigma$. The result is that

$$
g_{j}(V z) \leq K_{5} v^{\delta(\alpha+k / 2)} .
$$

Following Hecke, we can now easily complete the proof of the Theorem in the cuspidal case. For we then have $\delta=0$, so that $g_{j}(\tau)$ is bounded in $\mathcal{H}$ by (14). Hence,

$$
\left|F_{j}(\tau)\right|=y^{-\sigma} g_{j}(\tau)=O\left(y^{-\alpha-k / 2}\right)
$$


and a standard argument (e.g., [R, pp. 128-130]) yields $a_{n}(j)=O\left(n^{k / 2+\alpha}\right)$ as desired. In case $\delta=1$ we use the argument of Lemma 8 in [K2] to obtain

$$
v=\operatorname{Im}(z) \leq \operatorname{Im}(M z)+K_{6} \operatorname{Im}(M z)^{-1}
$$

for all $M \in \mathcal{M}$ and a constant $K_{6}$ independent of $M$. Then if $\tau=V z=$ $T^{n} M z$ as before, we obtain

$$
v \leq y+K_{6} y^{-1}
$$

so that with (14) we get

$$
\left|F_{j}(\tau)\right|=y^{-\alpha-k / 2} g_{j}(\tau) \leq K_{5}\left(1+K_{6} y^{-2}\right)^{\alpha+k / 2}
$$

and thus

$$
\left|F_{j}(\tau)\right|=O\left(y^{-k-2 \alpha}\right) .
$$

Again it is a standard consequence of this estimate that $a_{n}(j)=O\left(n^{k+2 \alpha}\right)$. This completes the proof of the Theorem.

4. Further comments. We make some further observations about vector-valued modular forms. It follows from (15) that if $k+2 \alpha<0$ then $F_{j}(\tau)=0$. Bearing in mind that $\alpha$ depends only on the relevant representation of $\Gamma$, we obtain

Lemma 4.1. Fix a representation $\varrho: \Gamma \rightarrow \mathrm{GL}(p, \mathbb{C})$. There is a real number $\beta$, depending only on $\varrho$, such that if $(F, \varrho)$ is a vector-valued modular form of weight $k<\beta$, then $F=0$.

The previous comments show that we may take $\beta=-2 \alpha$. We do not generally know the best possible value for $\beta$, which may well be 0 . For that matter, it is possible that the main Theorem holds true with $\alpha=0$. It is well known that if we take $\varrho$ to be a 1 -dimensional representation (i.e., forms on the full modular group), or more generally a unitary representation (essentially the case of classical modular forms on a subgroup of finite index, cf. [S]) then indeed we may take $\alpha=\beta=0$.

The nature of vector-valued modular forms of weight 0 is also of interest. We note that the existence of nonconstant (entire) vector-valued modular forms of weight 0 is established in $[\mathrm{KM}]$, though of course this cannot hold in the classical case. The following more specialized result finds application in $[\mathrm{DM}]$. See $[\mathrm{KKP}]$ for related results in the classical arena.

LEMMA 4.2. Let $(F, \varrho)$ be a vector-valued modular form of weight $k$ and assume that each of the Fourier coefficients $a_{n}(j)$ of each of the component functions $F_{j}(\tau)$ is a nonnegative real number. Then the following hold:

(a) If $k=0$ then each $F_{j}(\tau)$ is constant.

(b) If $(F, \varrho)$ is cuspidal then $F=0$. 
Proof. The fact that the derivative of a (classical, entire) modular form of weight 0 is a cusp-form of weight 2 continues to hold for vector-valued modular forms. Consequently, part (a) of the lemma follows from part (b). As for (b), observe that the nonnegativity of Fourier coefficients implies that the function $F_{j}(i y)$ is nonnegative and increasing for $y \rightarrow 0+$. On the other hand, consideration of $\left.F_{j}\right|_{k} S(i / y)$ leads to an equality

$$
F_{j}(i y)=i^{k} y^{-k} \sum_{n>0} b_{n} e^{-2 \pi n /(N y)}
$$

for some coefficients $b_{n}$, and this tells us that $F_{j}(i y)$ vanishes as $y \rightarrow 0+$. We conclude that $F_{j}(\tau)$ vanishes on the positive imaginary axis, and hence is necessarily 0 .

\section{References}

[DLM] C. Dong, H. Li and G. Mason, Modular-invariance of trace functions in orbifold theory and generalized Moonshine, Comm. Math. Phys. 214 (2000), 1-56.

[DM] C. Dong and G. Mason, Rational vertex operator algebras and the effective central charge, preprint QA/0201318.

[E] M. Eichler, Grenzkreisgruppen und kettenbruchartige Algorithmen, Acta Arith. 11 (1965), 169-180.

[K1] M. Knopp, Modular Functions in Analytic Number Theory, 2nd ed., Chelsea, New York, 1993.

[K2] -, Some new results on the Eichler cohomology of automorphic forms, Bull. Amer. Math. Soc. 80 (1974), 607-632.

[KKP] M. Knopp, W. Kohnen and W. Pribitkin, On the signs of Fourier coefficients of cusp-forms, to appear.

[KM] M. Knopp and G. Mason, Generalized modular forms, J. Number Theory 99 (2003), 1-28.

[L] J. Lehner, A Short Course in Automorphic Forms, Holt, Rinehart \& Winston, New York, 1966.

[R] R. Rankin, Modular Forms and Functions, Cambridge Univ. Press, 1977.

[S] A. Selberg, On the estimation of Fourier coefficients of modular forms, in: Proc. Sympos. Pure Math. 8, Amer. Math. Soc., Providence, RI, 1965, 1-15.

[Z] Y. Zhu, Modular invariance of characters of vertex operator algebras, J. Amer. Math. Soc. 9 (1996), 237-302.

Department of Mathematics

Temple University

Philadelphia, PA 19122-6094, U.S.A.
Department of Mathematics

University of California

Santa Cruz, CA 95064, U.S.A.

E-mail: gem@cats.ucsc.edu

Received on 21.1.2002

and in revised form on 11.3.2003 